Historic, archived document

Do not assume content reflects current scientific knowledge, policies, or practices. 



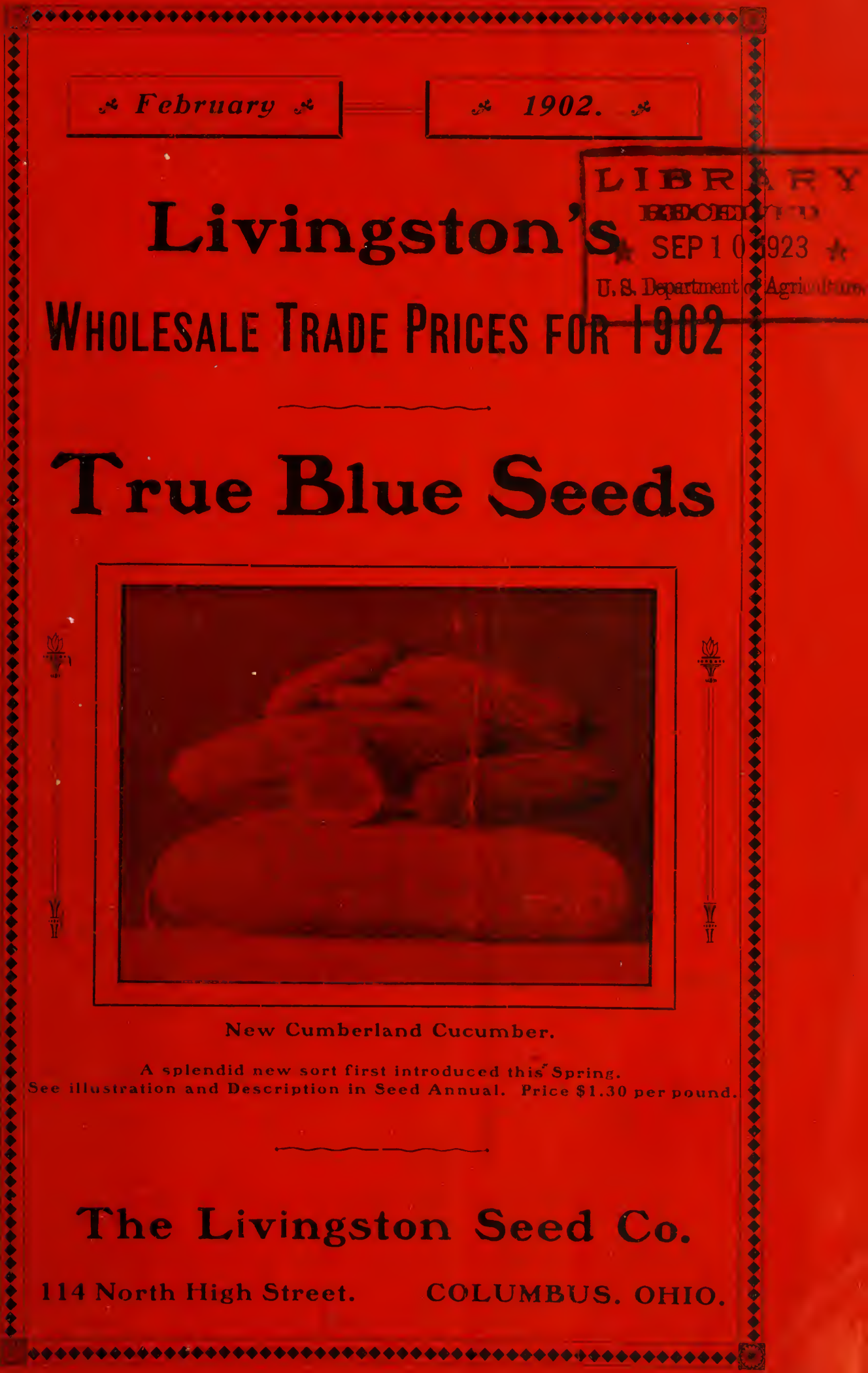




\section{To Dealers}

COLUMBUS, OHIO, February, 1902.

The quotations in this Trade List are intended for Dealers Only. We expect to bill at prices given as far as possible, but should any of our stocks run short we reserve the right to decline orders or advance price.

We do not put out Vegetable Seeds on Commission.

Transportation At the prices quoted customers must pay cost of forwarding. When desired we will send by mail, but when this is done postage will be added to the invoice at the rate of eight cents per pound. Quarter pounds will be supplied at pound rates; quarter bushels at bushel rates.

Cartage, Etc. We make no charge for cartage, boxes, barrels or packing. Bags only are charged for, and at cost, which is from 13 to 15 cts. each.

Terms All general bills of Garlen Seeds are payable June 1, 1902, without reference to date of purchase; or a discount of one per cent. a month will be allowed on all payments made before June 1. Bills to parties buying special items only, are due in 60 days. Oniou Sets, Potatoes, etc., are net cash. Unknown correspondents desiring credit must give satisfactory reference."

Seeds in Packets, Etc. Which cannot be billed at the low bulk prices given in this Bulk Wholesale List, will be inyoiced at following discounts from prices in our Retail Catalogue:

All Seeds in Packets, except those herein quoted, at 50 per cent. discount. 50() packets or more at 60 per cent. discount.

Seeds by the ounce, pint or quart, $: 331 / 3$ per cent. discount.

Potatoes by the pound, or Potato Eyes, 4l) per cent. discount

Plants in collections, 11 per cent discount.

Books on Garden Topics, 2" per cent. discount.

At these liberal rates of discount the goods must travel at the purchaser's expense, and if by mail, postage will be added to the invoice.

Special Notice.

We do not supply "cheap seeds," and thęrefore do not try to compete in price with those who handle them. We furnish the Trade exactly the same quality of seeds that we send to our best Market Gardencrs. They are grown either by ourselves or subject to our inspection; hence we feel no hesitancy in saying that they are the purest and best to be found. At the same time, however. it must be distinctly understood, since the success of a crop is dependent upon so many contingencies, as condition of soil, mode of planting, climate, etc., that we cannot warrant our Seeds or hold ourselves responsible for any loss or damage arising from any failure thereof.

Address all Orders and Inquiries:

\section{The Livingston Seed Co.}

Seed Growers; Merchants and Importers;

Successors to

COLUMBUS, OHIO.

A. W. Livingston's Sons,

RETAIL STORES AND OFFICES: 114 North High Street.

WAREHOUSES: 11 to $17 \mathrm{E}$. Long St., and Lane Ave. Farm. 


\section{THE LIVINGSTON SEED CO.'S}

\section{TRADE

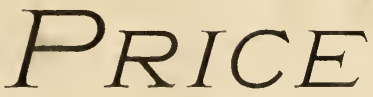 \\ LIST}

\section{PLEASE USE OUR “CIPHER" WORDS IN TELEGRAMS.}

$\mathrm{O}$

UR customers will find a Telegraphic Code word printed opposite each article, enabling them to order by wire at comparatively little expense For example: "Five Baneful" would mean five bushels Extra Early. Round Pod Valentine Beans; "Twenty Target" would mean twenty pounds Livingston's Beauty Tomato. The additional word, "Freight," "Express," "Mail," would designate the mode of shipment.

\section{ASPARAGUS.}

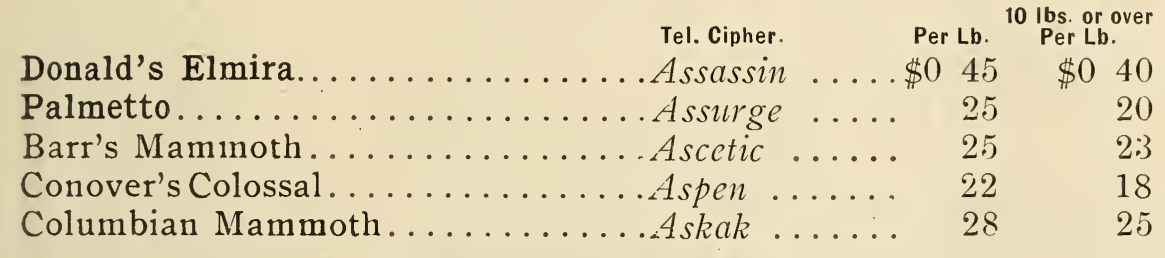

\section{ASPARAGUS ROOTS.} CHOICE TWO YEAR OLD STOCK.

Palmetto..................Arson

Per 1000

425

Conover's Colossal..............Article

Barr's Mammoth..............Armor

Donald's Elmira ...............Arbest

\section{BUSH BEANS, Wax or Yellow Podded.}

Livingston's New Pencil Pod Wax...Beaten ...... 500

Extra Early Valentine Wax.........Berth ...... 450

Currie's Rust Proof Wax..........Believe ..... \& 00

New Round Pod Kidney Wax..... . Bibul ...... 550

Extra Early Refugee Wax......... Berutch .... 425

Scarlet Flageolet Wax..........Bibac ......4 45

Perfection Wax................Betray ..... 475

Improved Golden Wax, Grenell's....Besiege ..... 400

New Prolific Black Wax...........Betroth ..... 425

White Seeded Wax................Bilber 


\section{BUSH BEANS, Green Podded,}

Extra Early Round Pod Valentine... Bancful .....\$3 25 Livingston's Round Six Weeks......Baker ...... 325 Extra Early Mohawk............Badness ..... 325 Early Long Yellow Six Weeks..... Bade ....... 325 Extra Early Refugee............Balcful ..... 325 White Seeded Valentine..........Barol ....... 450 Best of All................Baby ...... 400 Refugee, or 1000 to I..........Barrack .... 325 Dwarf Horticultural............Bandy ..... 4400 New Giant Stringless Green Pod....Bang ...... 350 White Kidney, or Royal Dwarf......Battes ..... 325

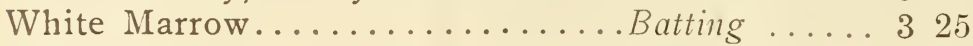

White Navy.................Bauble ...... 325

New Stringless Green Pod.........Bached ..... 350

Burpee's Bush Lima............. Billow ...... 600

Dreer's Bush Lima ..............Binder ...... 625

Henderson's Bush Lima..........Binmac ..... 525

New Wonder Bush Lima

Becker

650

0 bus. or over Per bu.

$\$ 300$

300

300

300

300

425

375

300

375

325

300

300

300

325

575

600

500

BEANS, Pole or Corn.

Livingston's New Early Dawn Lima. Banker

Ford's Mammoth Podded Lima..... Bosheit

700

625

Siebert's Early Lima.

Barbour

Early Golden Cluster Wax ......... Bluster

625

(5) 00

Lazy Wife's.

Boggle

Large White Lima.

Botarge

Dreer's Improved, or Challenger LimaBoscage

White Crease Back, or Fat Horse... Bootl

575

5) 50

Jersey Extra Early Lima. .

Bosom

600

King of the Garden Lima. .

Botanise.

575

Early Dutch Case Knife.

Blighted

450

Speckled Cranberry, or Horticultural. Boaster

Southern Prolific; True Round Pod..Bony ...

450

450

Small Lima, or Sieva............ Bottle

$5 \quad 50$

Kentucky Wonder, or Homestead ...Bodkin

550

Red Speckled Cut Short, or Corn Hill. Bonmot

Livingston's Royal Corn Bean ......Boyer

Powell's Prolific Snap

\section{GARDEN BEET.}

New Crosby's Egyptian(Home Grown)Bracelet 750

600

$5 \quad 75$

600

525

$5 \quad 50$

525

550

425

425

425

525

525

\section{(50.....}

Per lb. 10 lbs. or over

50

45

35

30

30

25

Extra Early Egyptian Turnip....... Brained ......

Arlington Favorite Blood Turnip....Braggard ....

Early Bassano................... Bragger .....

32

36

28

24

Dewing's Improved Blood Turnip. . . Rract . .

Bastian's Early Blood Turnip....... Erabback .....

Half Long Blood, very choice ...... Brabbade .....

Long Dark Blood Red............Branch ......

Edmand's Early Blood Turnip...... Brain .......

Lentz Extra Early Blood Turnip.... Brasen

Detroit Dark Red Turnip ..........Brackish . . 
MANGEL WURZEL.

Mammoth Long Red.

Tel. Cipher.

10 Ibs. or over

Mammoth Red Globe. Bricr Per 16. er Ib.

Golden Tankard

Brisk

$\$ 0 \quad 13$

16

14

18

Champion Yellow Globe..........Brefer

Yellow Leviathan

Brock

\section{SUGAR BEETS.}

Lane's Imperial.

French White Red Top..........Broncho

Vilmorin's Imperial

Broanie

\section{BROCCOLI.}

Purple Cape.................Bursche

\section{BRUSSELS SPROUTS.}

Improved Dwarf.............. Byback

\section{CABBAGE.}

Henderson's Early Spring ........Carly ...... 2 25

Earliest Express, or New WonderfulCauser ...... 100 All Head Early................. Caravas ..... 210

Large Charleston Wakefield........Carbine ..... 175

Select Extra Early Jersey Wakefield.Carun ....... I 60

Select Henderson's Early Summer ..Centime ..... 160

Early Flat Dutch .............Carrom ..... 160

Early Winningstadt, select strain.... Castel ...... 80

Fottler's Improved Brunswick. ... . Cavity ....... 110

Henderson's Succession............ento ....... 175

All Seasons, or Vandergaw........Capture ..... 160

Danish Ball Head, Direct from Den-

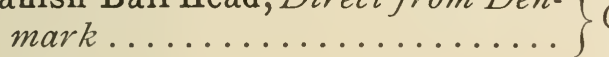

The Lupton.................Chief ...... 125

Autumn King, or World Beater.....Choker ...... 150

Chase's Excelsior, choice second early'Carbisk ..... 150

Large Late Flat Dutch ...........Chandler ..... 125

Livingston's New Ideal Winter...... Centrip ..... 150

Burpee's Surehead ............. Chintz ...... 140

Large Late American Drumhead ....Chancel ...... 125

Early Louisville Drumhead.... . . . Chare ... . . . 120

Marblehead Mammoth..........Charity ..... 125

Mammoth Rock Red........... Chariot ..... 150

Improved American Savoy......... Cheek ...... 125

Livingston's Select Premium Flat? Dutch 
Livingston's Early Market

Tel. Cipher.

10 Lbs or over

Guerande, or Ox Heart.... .

Clarion

Per lb. Per lb.

Earliest French Forcing, or Gold BallClamor $\$ 0 \quad 60$

$\$ 0 \quad 55$

Intermediate Red.

. Clear

70

65

Early Scarlet Horn (stump rooled)... . Clamber

55

50

Improved Long Orange.

Cleve

65

60

Danver's Half Long.

Classic

55

Nantes Eariy Half Long Scarlet. . . . Clipper

Chantenay.

Clabbisk

Improved Short White (for stock)...Ciement

White Belgian (for stock).

Cleri'

\section{CAULIFLOWER.}

Livingston's Earliest.

Cicago

Per oz. Per lb

Henderson's Early Snow Ball....... Cicame ..... 160

2500

Extra Early Dwarf Erfurt......... Cicaffle

Half Early Paris, or Nonpareil..... Cibare .

1800

Lenormand's Short Stem...

Cidead

$6 \quad 50$

Large Late Algiers

Cicamord

Veitch's Autumn Giant

Cideone

\section{CELERY}

New Golden Rose, Self Blanching...Comate ...

Giant Pascal..................Cohort ...... $\quad 90$

White Plume, very choice strain ...Compound ... 1 2.j

Golden Self Blanching ..........Coiner ...... 340

Evan's New Triumph........... Combine .... 150

New Rose.......................... 125

Dwarf Golden Hearted

Codger

Giant White Solid

Commerce

Boston Market

Coif .

Kalamazoo ... .

Cobble

Giant Golden Heart

Colly

Perle Le Grand

Coliere ...... 100

Pink Plume.

Comming ... 160

Flavoring (old seed).

Century

125

Giant Prague Celeriac

Comate

Conch ...... 100

Ibs. or over Per Ib, 200

\section{COLLARDS}

True Georgia................Condole ..... 50

\section{CORN SALAD}

Large Broad Leaved.............Condome.....

\section{CORN, Garden Varieties.}

Kendal's Early Giant...........Comflux .... 260

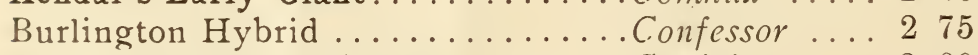

Extra Early Red Cob Cory..........Conjoin ...... 260 
CORN, Continued.

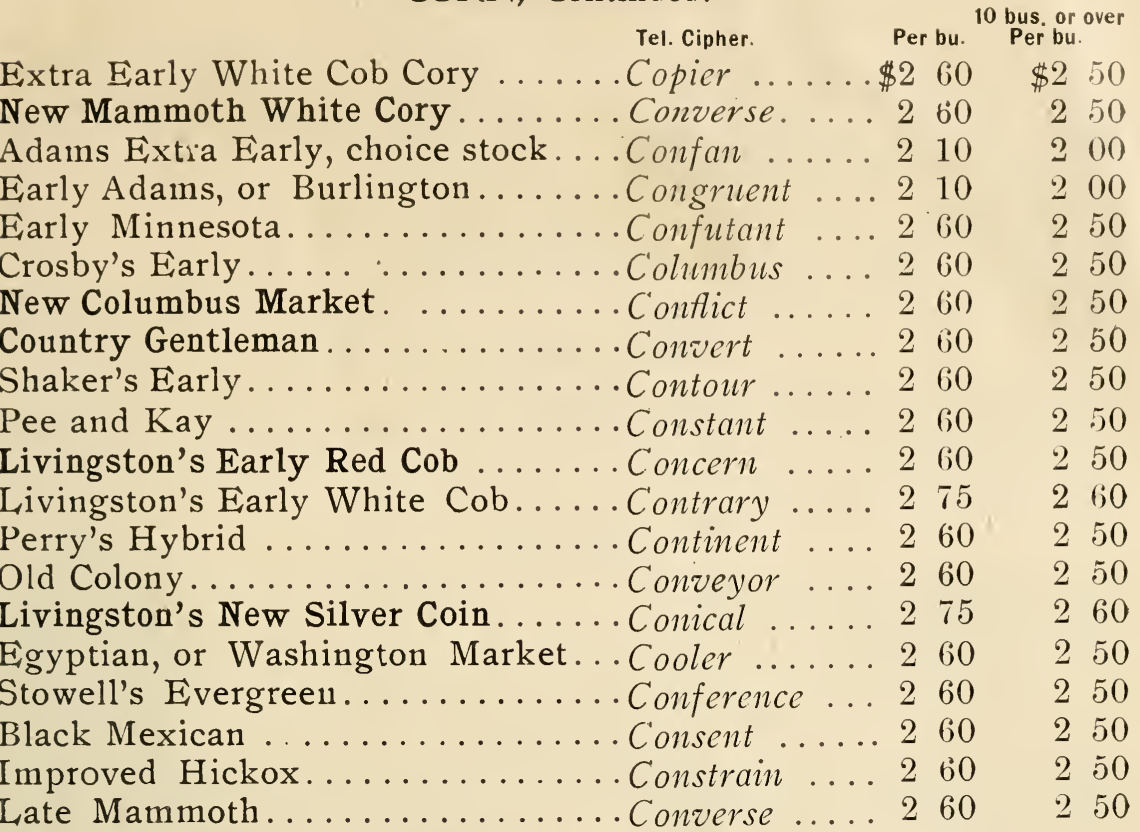

CORN, Field Varieties. $\left.\begin{array}{c}\text { Livingston's New Early Golden Sur- } \\ \text { prise ....................... }\end{array}\right\}$ Curden .... 50

True Clarage Yellow............Cordate ..... 140

Mammoth White Dent..........Coupon ..... 140

True Yellow Leaming........... Corumule ..... 140

White Cap Yellow Dent.......... Corumack .... 140

New White Cob Early Dawn....... Cornice ..... 1.50

\section{CRESS.}

Extra Curled (Pepper Grass)........ Cuniform .... 30

True Water, 20c per oz..........Curacva .... 225

Upland, $30 \mathrm{c}$ per oz............. Curracy ..... 300

\section{CUCUMBER.}

New Cumberland.............Coalport .... 130

Livingston's New Emerald......... Cracker ...... 40

Livingston's Evergreen Pickle......Crossrođd .... 30

Hill's Forcing White Spine........ Crocket ..... 35

Nichol's Medium Green ........... Crucial

Arlington Improved White Spine.... Crosier

Livingston's Select Extra Early

White Spine.

Peerless White Spine...........Cruelty

Green Prolific or Boston Pickling.... Croaket

Jersey Pickle................. Crossbol

Early Green Cluster............. Cretin ....... 


\section{CUCUMBER--Continued.}

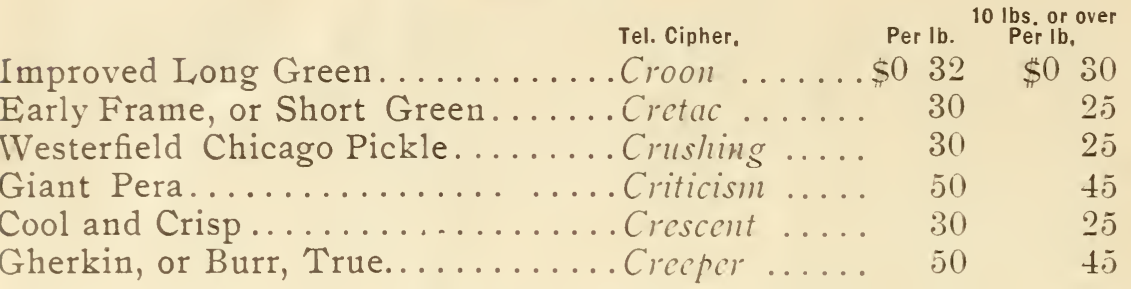

\section{EGG PLANT.}

Livingston's M a m moth Purple?

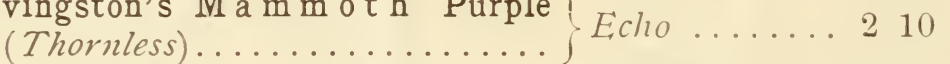

200

New York Large Purple..........Easeling . .

$200 \quad 190$

\section{ENDIVE.}

Fine Green Curled Winter... ......Embogue .... 80

Ever White Curled.............Elcctric ..... 80

Broad Leaved Batavian (Escarolle).. Egotist

KALE.

Siberian, or Dwarf German Greens..Burgher ..... 35

Dwarf Green Curled Scotch......... Bungling ... . . j0

\section{KOHL RABI.}

Early Purple Vienna.

Kíblap

$\begin{array}{llll}1 & 60 & 1 & 50\end{array}$

Early White Vienna Kolpic

\section{LET TUCE.}

New Wonderful, mammotl size; fineLitanl' ....... 80

Livingston's Crisp as Ice....... . Leaguer

Grand Rapids, extra fine for forcingLeggin

Improved Hanson, select stock....... I.egume

Big Boston.......................................

Denver Market.

White Seed Tennis Ball, (Boston)

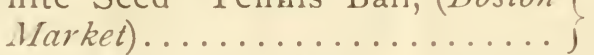

Black Seed Tennis Ball...........Lodgment ....

Defiance Summer. ............... I cssor .......

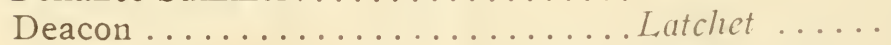

Silver Ball..................... Jubule ......

Black Seeded Simpson, Select....... Lanaman .....

Prize Head................... I.instock .....

Early Curled Simpson.............Loburst ......

Tilton's White Star.............. Iogline .....

Early White Cabbage, or Phil ButterLazar .......

Celery Lettuce, Trianon Cos........Logman .....

Improved Mammoth Salamander....Letting ....... 


\section{LEEK.}

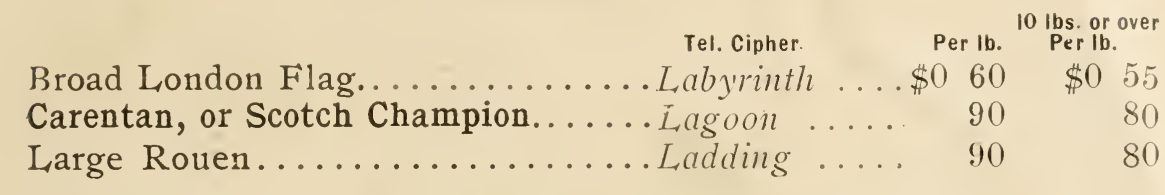

\section{MUSKMELON.}

New Paul Rose.............. Madcap ..... 40

Livingston's New Tip Top.........Mclody ..... 40

Columbus ...................Majesty .... 30

Living'ston's Market, crop very shortMarrow

The Rose Netted Gem............II grose

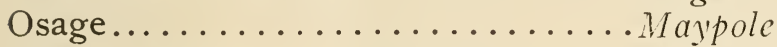

Emerald Gem................Mallard

Extra Early Green Citron..........Malleg

Champion Market.............Maihem

Extra Early Hackensack.......... Maniple

Perfection (The Princess).........Meantime

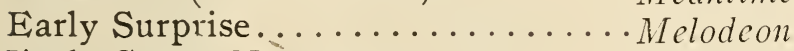

Early Green Nutmeg...........Malario

Skillman's Fine Netted.........................

Winter Pineapple..............Mcmorize

Hackensack, or Turk's Cap.........Manor

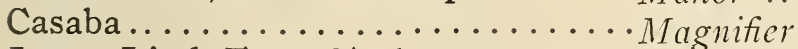

Jenny Lind, Extra Early............. Marker

Miller's Cream.

Massive

Bay View.

- Magic

Acme; or Baltimore Nutmeg.......Madam

Burpee's Netted Gem.............Mattock

Banana

- Madman

Banquet.

Magian

Shumway's Giant..

Medicine

Early Grand Rapids.............Meteornc.....

Beck's Triumph.......................................

Improved Anne Arundel . . . . . . . Magnet

Chicago Market...............Maiming

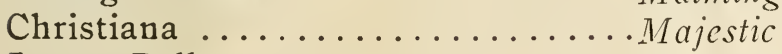

New Osage Gem.

Maximi

\section{WATERMELON.}

Livingston's Nabob.............Modern ......

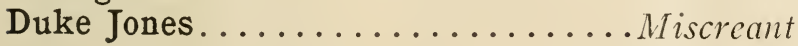

Livingston's Gragg. . . . . . . . . . . Milage 


\section{WATERMELON--Continued.}

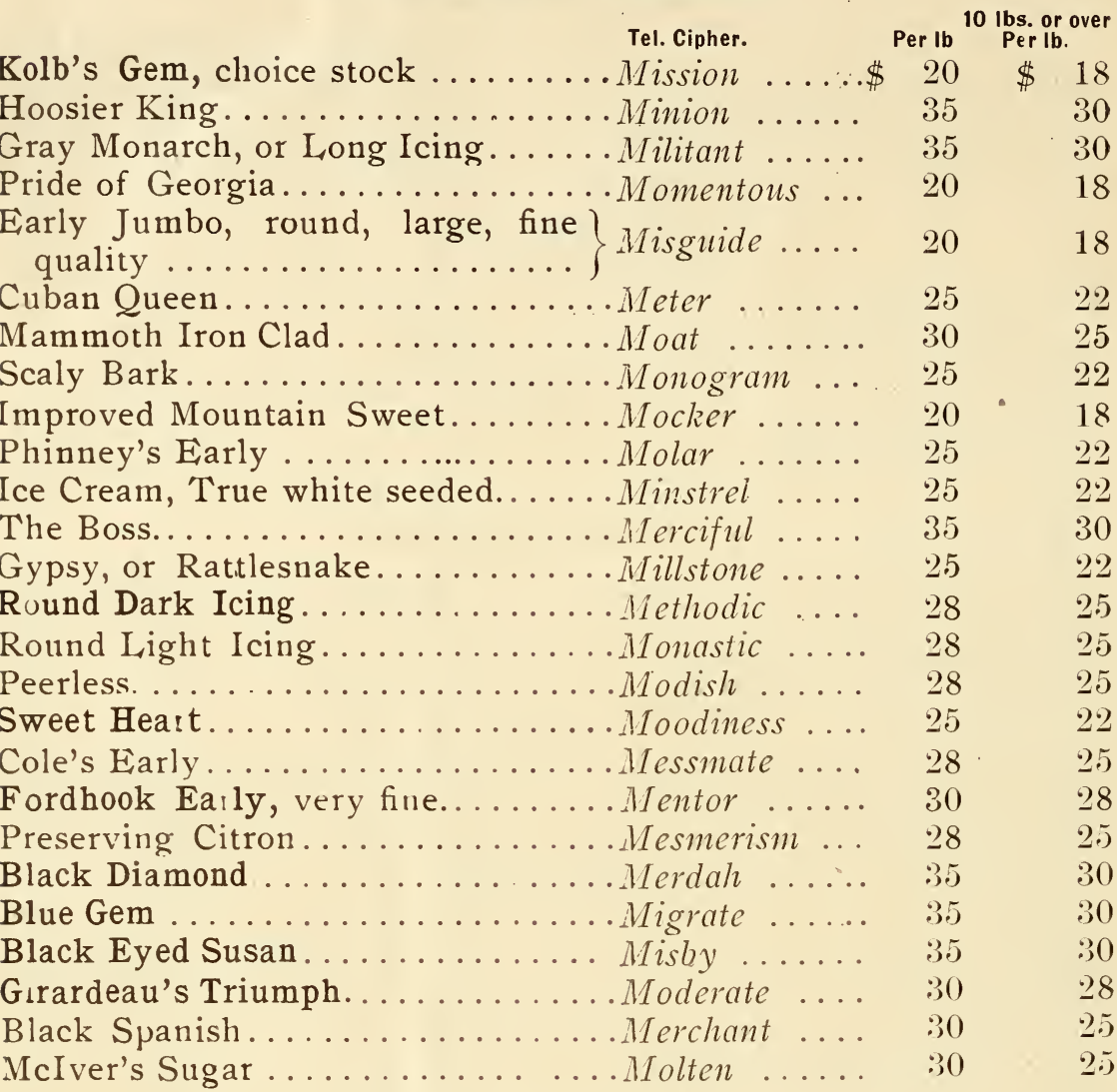

\section{ONION.}

Livingston's Brown Beauty........ Orton ...... 150

Extra Early Red Flat........... Oddity

Extra Large Red Wethersfield...... Omous

Improved Yellow Globe (Under) wood Strain)..

Obvite ...... 110

Southport Red Globe, our own growthoracle

Yellow Danvers................ Oching

Select Yellow Danvers Globe ?

$$
\text { (Eastern Grown) .......... }
$$

Outig ...... 90

Ohio Yellow Globe, our own growth. Ophide ..... 110

Philadelphia Yellow Strasburg, for setsOposs

Large Yellow Dutch, for large onions Onset

Southport Yellow Globe, Conn.GrownOral

Mammoth Prizetaker........... Opake . .

Southport White Globe, Conn.GrownOrdain ..... 160

White Portugal, for large onions.... Ouchab .... 160 


\section{ONION-Italian Varieties.}

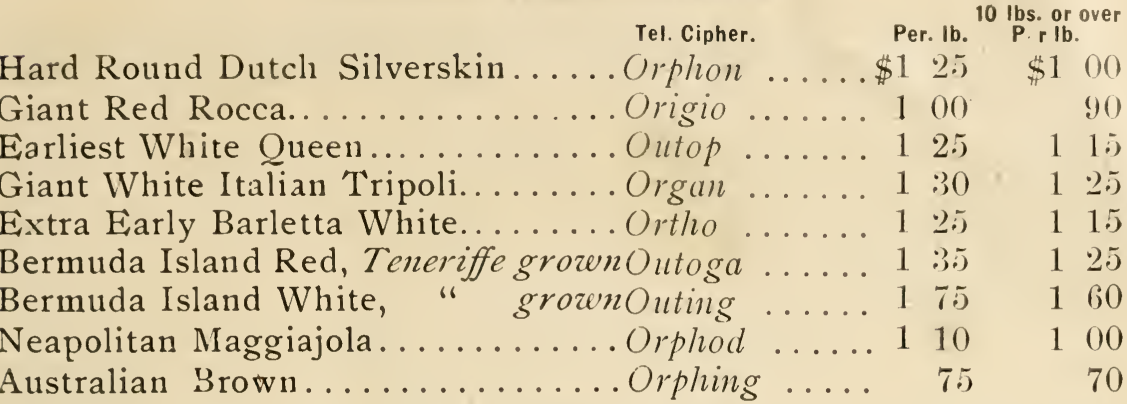

\section{MUSHROOM SPAWN.}

Best English Milltrack.......... Mumbler ..... 10

\section{MUSTARD-For Growing.}

White English, or London.........Mourner ..... 12

Southern Giant Curled...........Motto ...... 30

New Chinese.

Morion

\section{ONION SETS.}

Red Bottom.......... Orabog .....32 lbs. to bu.

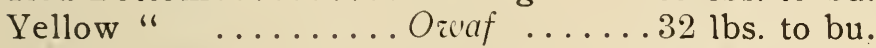

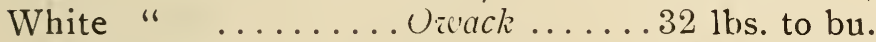
White Multiplier...... Orvacked ....28 lbs. to bu. Egyptian, or Perennial.Orup ......28 lbs. to bu. Yellow Potato, True... Oruakup .....32 lbs. to bu. Top or Button........ Occur ......28 lbs. to bu.

White Velvet, extra fine.......... Oblate ...... Tall Green...

Oath

\section{PARSLEY.}

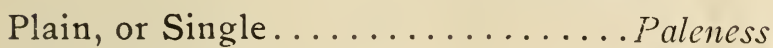

Fine Double Curled..............Pagoda

\section{PUMPKIN.}

Livingston's Thanksgiving........Pouridge.....

Connecticut Yellow Field ......... Polymp .......

Calhoun...................Polygram

Mammoth Potiron.

Popery

Large Cheese.................Pondal

Tennessee Sweet Potato..........Poser .........

Japanese Pie................Pompilo .....

Mammoth Tours............. Poplit 


\section{POTATOES---Northern Grown.}

Our barrels contain $23 / 4$ bushels. We deliver Potatoes to the transportation companies carefully packed; after that our responsibility ceases. Purchasers must take all risk from freezing or heating. We make no charge for barrels or cartage. Prices quoted are F. O. B Columbus. Prices subject to market changes.

Livingston's Banner........... Pict $y^{\prime} \ldots \ldots \ldots \ldots \ldots \$ \$ 400$

Seneca Beauty...............'llenary ......... 400

Extra Early Ohio............Piscary ........ 400

The Livingston.............Pikestati ....... 400

PARSNIP, American Grown.

New Zimpfer.

Tel. Cipher.

Selected Hollow Crown.

Palmy

Per lb. 10 lbs. or ove

$\begin{array}{llllll}\$ 0 & 60 & \$ & 0 & 50\end{array}$

$20 \quad 18$

\section{PEPPER.}

Improved Thick Long Red.........Pholly

Red Cherry.................Phl'sical

Sold out.

Mammoth Ruby King ............Ptiilomel ... $150 \quad 140$

Sweet Mountain................ Picaroon ..... $14010 \quad 130$

Bull Nose, or Large Bell..........Pctrel ...... 1 to $1: 30$

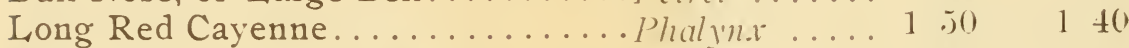

Red Chili.................Picket ..... 1 .j0

Golden King, or Queen..........Pcaling .....2 00

Long Red Cardinal............Pallot ......200

PEAS.

\section{Livingston's Giant Podded Prolific ?} Sugar

Prosperity, or Gradus.

Livingston's First in Market.

Gregory's Surprise............. Parado.

Extra Early Alaska.............. Pannicr

First and Best.............. Fasturage

Improved Dan O'Rourke........... I'au'ner

American Wonder, true stock......Pantaloon

McLean's Little Gem............Pediment

Premium Gem

Pencern.

Philadelphia Extra Early...........Pcnalty

Nott's Excelsior.

Pellet

Juno.

Peaceful

Shropshire Hero..............Penurv'

Carter's Daisy................Parboi

Heroine.

Paíer

Horsford's Market Garden......... Pavilion

McLean's Advancer

Pedestal

Pride of the Market.

Penchiant

Telephone, Select stock.

Perfidous

Long Island Mammoth. . .

Pedantry

Improved Stratagem, very choice... Pcrcolate

Abundance.

Pantry

Papa
Per bu 10 bus. or over

Sold out.

Sold out

47.5

42.5

400

475

475

475

(5) 00

575

5) 75

50

$5 \quad 50$

570

47.5

(5) 25

3) 50

32.5

3) 75

700

725

3.50

375

3 75

400

Sold out

4 2.j

400

Sold out

Sold out

450

425

3) 75

3.50

3) 7 
Champion of England. . Per bu. Per bu.

Yorkshire Hero. Pareni $\$ 375$ $\$$ ऽ 50

Melting Sugar. Pergolt

Black Eye Niarrowfat hand picked...Pantomime ... 2 50

White Marrowfat, hand picked .....Perforce .... 250

235 Canadian Field, No. 1, white.......Pathos

Market price.

\section{RADISH.}

Turnip, Forcing Scarlet...........Rattle

Per lb. 10 Ibs. or more

" " $\left.\begin{array}{c}\text { Rosy Gem......... Reclaim ...... } \\ \text { (Fireball)......... }\end{array}\right\}$ Rayden .....

Rebec.

Early Scarlet.................Ranson

Scarlet, white tipped......Regara

Yellow Summer.

Regress

New Golden Dresden......Rayman

Large White Summer...... Reagable .....

Golden Gilobe............Raymel ......

Gray Summer...........Razure

Olive, Forcing V'ick's Scarlet Globe.. Reccruit

New Leafless........ Ramage

White Rocket........Receptive ....

“ French Breakfast..........Rayful .....

Half Long, Deep Scarlet.......... Reach ......

Long, Cincinnati Market..........Recharge ....

Scarlet Short Top.........Rapacious ....

" Brightest Scarlet White TippedReally ...... .

New White Icicle..........Rascal .......

" Livingston's Pearl..........Rebound .....

" White Chartier............Raid .......

" Improved Chartier.........Reading ....

Summer, White Strasburg.........Refuple

" Giant White Stuttgart....Reform

Winter, California Mammoth......Regam

" China Rose............. Recital

Long Black Spanish........ Reality' .....

Celestial or White Chinese. Ragman .....

Scarlet Pamir..........Recess ......

Long White Spanish...... Rasping ....

Round Black Spanish......Reclose

\section{RAPE.}

Dwarf Essex.............. Racing .... 10

\section{RHUBARB.}

Large Victoria 
SALSIFY.

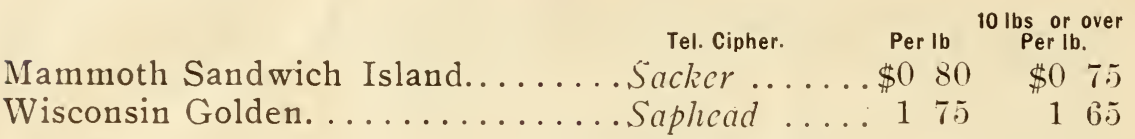

\section{SPINACH.}

Bloomsdale Savoy. ............. Sanscrit ..... 12

Best Prickly Seeded Winter........ Sapplic ......

Best Viroflay Thick Leaf......... Satole .... . . 12

Best Long Standing.............Sandal ...... 12

New Victoria................. Satrap ...... 12

Round Leaved Summer...........Salty' ....... 12

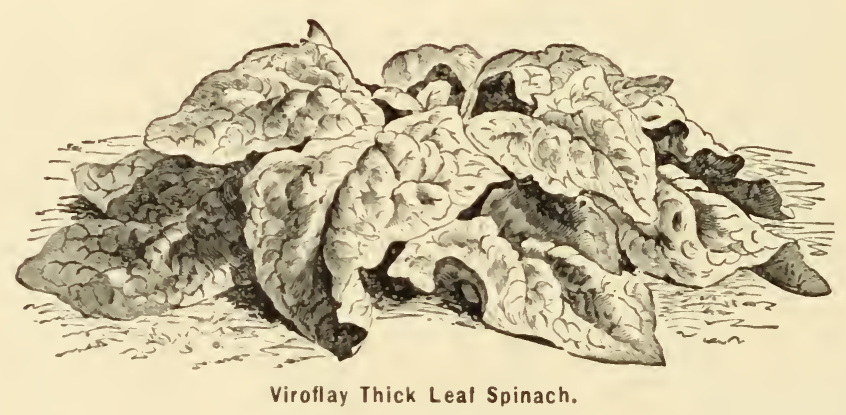

SQUASH.

Mammoth Whale..............Scholic ..... 1 2.)

Livingston's New Pie ...........Scroll ...... 40

New White Summer Crookneck...... Soldicr

Fordhook .................Sciatic . . .

Sold out

Mammoth White Bush Scallop......Scutcheon ... 35

Mammoth Yellow Bush Scallop or

Golden Custard............

Giant Golden Summer Crookneck. . . Scioptic .......

Golden Summer Crookneck.........Scotchman ...

Early White Bush Scallop..........Secular ......

Hubbard, choice stock ............. scoundrel . . .

Livingston's Improved Cushaw. . . . . Sclector . . . . . .

Essex Hybrid.......................

Boston Marrow................. Scarf .......

Colvin's Early Orange Marrow......Schemer . .

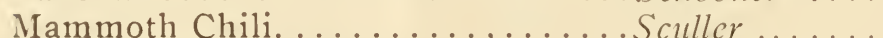

Chicago Warty Huobard.. ......... Scaffold 


\section{LIVINGSTON'S \\ C E L E B R A T E D \\ TOMATO SEEDS \\ FOR MERCHANTS \\ IN SEALED PACKAGES ONLY}

For the protection of the honest merchant and the grower who desires only true stock of our Tomatoes, we have for several years followed the plan of supplying Our Celebrated Varieties of Tomato Seeds in Sealed packages Only; aud hence every package of the Livingston varieties of Tomatoes grown at headquarters will be sent under our TRUE BLUE SEAL.

In 1870 we originated and introduced our Paragon, which was the first perfectly smooth red variety known. Five years later we introduced the Acme, the first perfectly smooth purple sort known. Since the advent of these sorts we have brought out consecutively the Perfection, Golden Queen, Favorite, Beauty, Potato Leaf, Stone, Royal Red, Aristocrat, Buckeye State, Honor Bright, Yellow Prince and Magnus. As originators and introducers of these sorts, we use the most assiduous care in growing the Seeds, and although these same varieties are extensively advertised by other dealers, yet we do not hesitate to claim superiority for our Headquarters' Strains.

\section{SPECIAL NOTICE}

We annually supply hundreds of merchants in all parts of the United States with our Tomato Seeds put up in packages for retailing. It is a highly satisfactory and successful method, because it affords a dealer's patrons positive evidence that he is selling the genuine article.

We put up the Seed in OUNCES, QUARTER-POUNDS and POUNDS, and each package bears our TRUE BLUE SEAL.

Packets for retailing at 5 and 10 cents are supplied to dealers at half price.

To those who have never handled our Seeds we respectfully suggest the propriety of trying this plan.

TOMATO---Livingston's Varieties. Tel. Cipher,
10 lbs or over Per lb Per lb.

Livingston's New Magnus........Theorb .....\$2 $50 \quad \$ 225$

Livingston's New Yellow Prince....T Telic ...... $200 \quad 190$

Livingston's Honor Bright.......Tennis ..... $160 \quad 150$

Livingston's Aristocrat..........Taproot ..... $250 \cong 25$

Livingston's Buckeye State.......Tarnish ..... $175 \quad 160$

Livingston's Royal Red...........Theatric ..... $140 \quad 135$

Livingston's Stone.............Thesis ...... $130 \quad 125$

Livingston's Potato Leaf. .........T'Cther ....... $150 \quad 140$

Livingston's Beauty............Target...... $150 \quad 140$

Livingston's Paragon............Terror ...... $140 \quad 135$

Livingston's Acme............Tapan ....... $150 \quad 140$

Livingston's Favorite............ T'cpidity ..... $150 \quad 140$

Livingston's Perfection........... Testan ...... 1400135

Livingston's Golden Queen. .......Templar ..... 160 T 150 


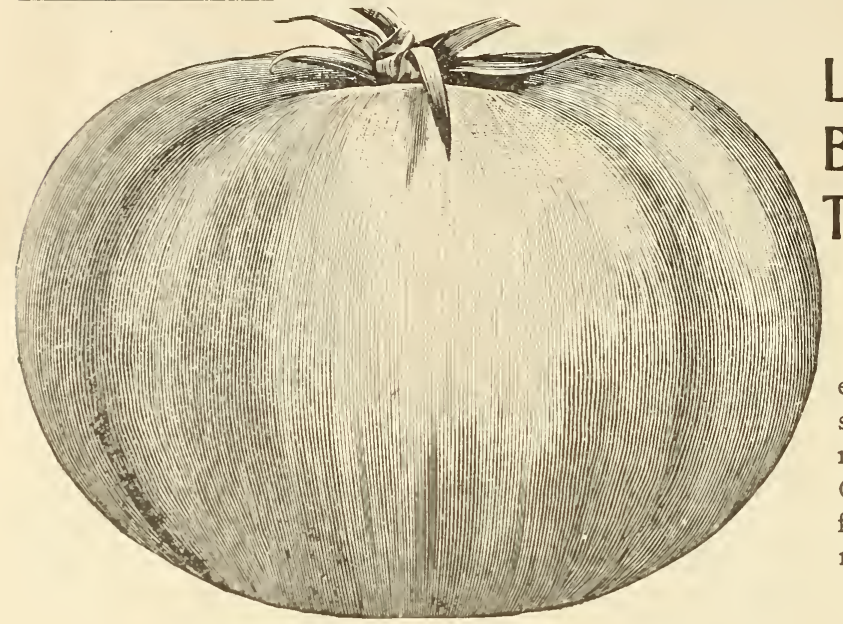

TOMATO-Continued.

\section{Livingston's Buckeye State Tomato.}

This is the largest fruited perfectly smooth PURPLE tomato in cultivation. One of the most satisfactory sorts for the merchant to handle.

Dwarf Champion............ Tauruls ....\$2 50

10 ibs. or over Yellow Husk................Tcnancy .... 175 Purple Husk...............Tcxthand .... 1 To Atlantic Prize-Very select stock... Tapster .... 1 2.5 Enormous............... Textile ..... 170 Plum Shape Yellow............Testimony ... 150 Matchless..................Termagan .... 140 Ponderosa................ Toaster ..... 300 Ignotum .................Tender ..... 125 Turner's Hybrid, or Mikado.......Thinker ...... 175 Improved Trophy.............. Theocracy ... 140 Essex Hybrid............... Tasting . .... 150 $\$ 240$ Purple Peach ................Tertial ..... 1 7.j Yellow Peach............... Thoral ..... 160 Early Ruby .................Tearless .... 1 75 Yellow Pear................Tegumen .... 175 Red Pear................. Terrific.... 175 Long Keeper, Thorburn's........T Tepor ...... 140 Cumberland Red..............Tomboy .... 140

Purple Top Strap Leaf.......... Tiptoc ....... 22

Purple Top White Globe......... Trode ....... 24

Seven Top................. Titmouse.... 20

Early White Flat Dutch.......... Thum . . . .

Early White Egg Shaped........ Token ..... 35

Amber Globe. ............... Thrice ...... 22

Large White Globe............ Timbrel ..... 20

Yellow Stone, very fine......... Topaz ...... 


\section{Genuine}

\section{Livingston's}

\section{* Beauty Tomato}

THIS is by all odds the most popular tomato in cultivation today among marliet gardeners. It is used in all sectionsnorth, south, east, west-with unfailing success. One would think that even such an old standby must suffer on account of the numerous introductions of new varieties, but on the contrary the genuine old Livingston Beauty is constantly growing in popularity-holding all its old friends and daily making new ones. There are about a dozen so-called new varietles on the market which are simply a poor strain of our time-honored Beauty. Dealers should buy their supply from us in order to be sure to get the genuine. We put it up in packets, ounces, quarter pounds and pounds at no extra expense to dealers and when handled in this way the merchant relieves himself from all responsibility and all chance of error by his clerks. Every package bears our True Blue Seal.

Price, see page 13.

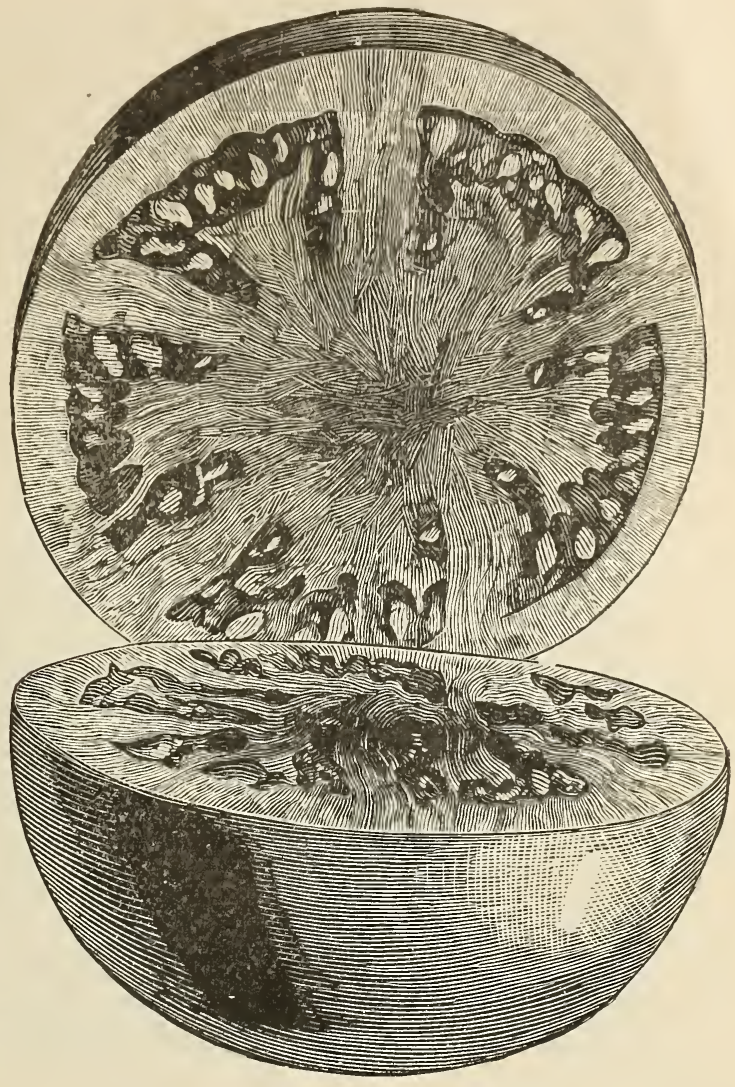

\section{GENUINE LIVINGSTON'S STONE TOMATO}

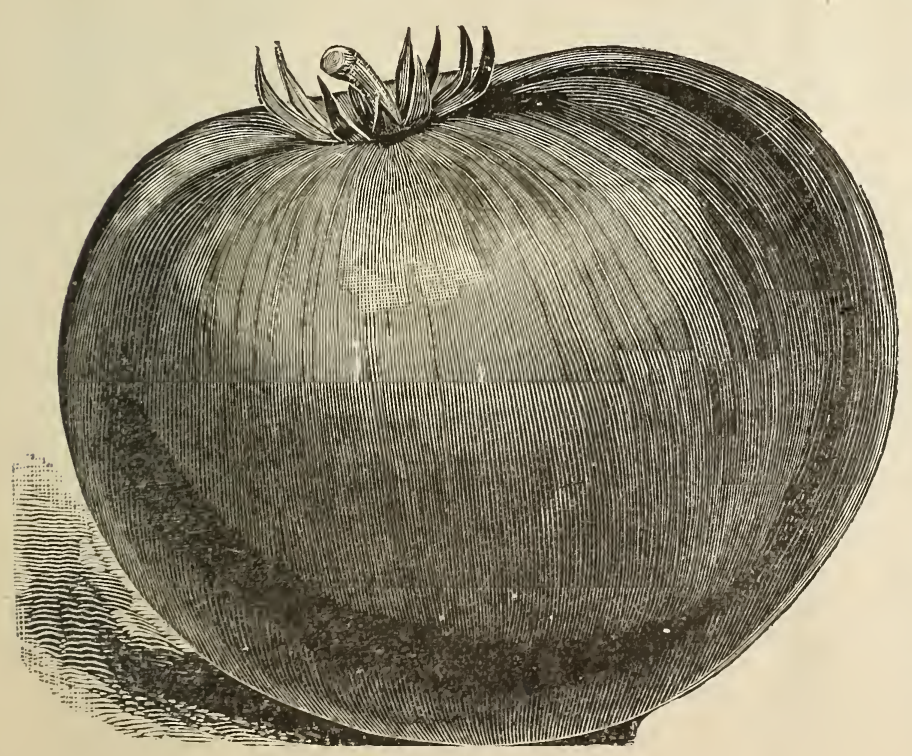

HIS great tomato, introduced by us only a few years since has headed the list of main crop red sorts from the beginning. As in the case of the Beauty (referred to above) dozens of imitations have been foisted upon the public and not a single advantage brought about by any of them. We have the GENUINE ORIGINAL Livingston's Stone and it is not equaled for main crop. It is of large size, perfectly smooth, and best table quality. The heaviest cropper known, being used almost exclusively by the largest growers evervwhere. It is perfectly solid, very meaty and has less seeds than any other variety of equal smoothness in cultivation. The Stone is still growing in favor year by year. Get our genuine seed in sealed packages and be safe. Your trade will grow and be permanent.

Price, see page 13 


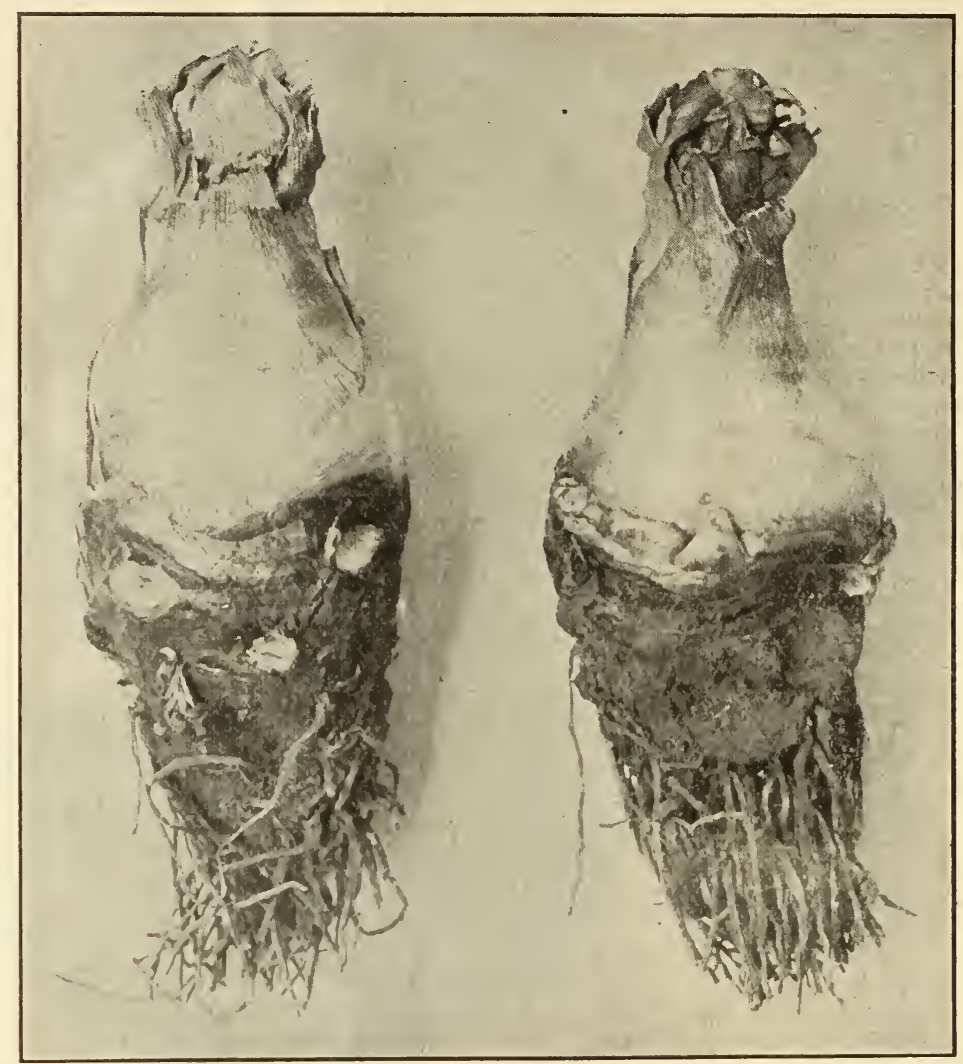

\section{BULBS.}

\section{Excelsior Pearl Tuberose.}

The crop of Tuberose Bulbs is very shot this zear and on account of drouth are hardly as large as usual. This will be true to all the Tuberose Bulbs in the market this spring Our stock is as fine as the best.

Price, First Size, $\$ 1.00$ per $100 ; \$ 8.00$ per 1000.

\section{Planet Jr. Implements.}

We handle these most excellent Tonls in large quantities and allow a discount of 25 per cent. from list prices. Also an additional discount of 5 per cent. if bill is paid within 30 days from date.

Tel Cipher. Price

No. 5, Hill and Drill Seeder....Fxactio. 1300

" 4, Comb, Hill and Drill

Seed and Cultivator
comolete.................. Choma . 1000

4. Comb, Hill and Driil

Seeder, as a Drill only..Chunni.. 800

“ 3, Hill and Drill Seeder. Chalant. 1000

“ 2, Drill Seeder.............Annexio 700

“ 2, Drill Seeder..............

vator. ….............. Annifer. $\quad 760$

“ 11, Nounle ivieoi Hoe.... Falsata $\times 50$

.. 12. Double Wheel Hoe.... Famis... 650

“ 13, Double Wheel Hoe....... Fana.... 450

“ 15, Single Wheel Hoe........ Evolito. 520

16, single Wheel Hoe...... Faleris. 550

" 17. Single Wheel Hoe ....Fallent . 475

" 18, Single Wheel Hoe....... Evulso.. 260

Grass Edger.................. Annuto. 480

12-Tooth Harrow and Cultiva-

tor, Plain..............Cantabo. 550

\section{BULBS.}

Caladium Esculentum.

(Elephant Ears)

Mealthy Bulbs Well Cured.

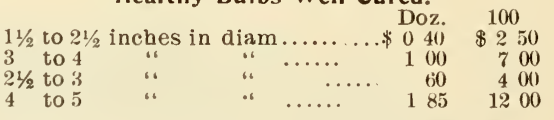

040
14250

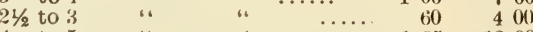

4 to 5 .

12-Tooth Harrow and Cultiva-

Tel. Cipher Price

tor, with Wheel ....A Arido... $6 \mathbf{5 0}$

12-Tooth Harrow and Cultivd-

tor, Complete............Bellosa. 800

No. 8. Horse Hoe Complete...... Chalseo 750

“9. Horse Hoe Complete ...... Farcino. 650

Firefly, Garden Plow .............Ansata 25

We will be pleased to send a Complete Catalogue of these too's, postpa $d$, on request.

\section{Paper Seed Bags.}

Per 100. Per 1000

No. 1, Coated, $2 \frac{1}{4} \times 3$ in., for Flewer Seeds .......

2, $23 / 4 \times 4$ in., for packets of Cabbage etc

" 3, $3 \frac{1}{4} \times 4 \frac{1}{2}$ in., for packets of Beets. Beans. etc.

"4. $3 \frac{3}{4} \times 5$ in., for ounces etc

$5,4 \frac{1}{2} \times 6$ in., for quarterpounds, etc........

“6, 51/4 $\times 8$ in., pint size......

“7, $6 \frac{1}{2} \times 9 \frac{1}{2}$ in. qu irt size.

“ $8,7 \times 10 \frac{1}{2}$ in, three pint size..
$15 \quad 110$

$18 \quad 140$

25200

$20 \quad 185$

$35 \quad 325$
$20-165$

$25-250$ 


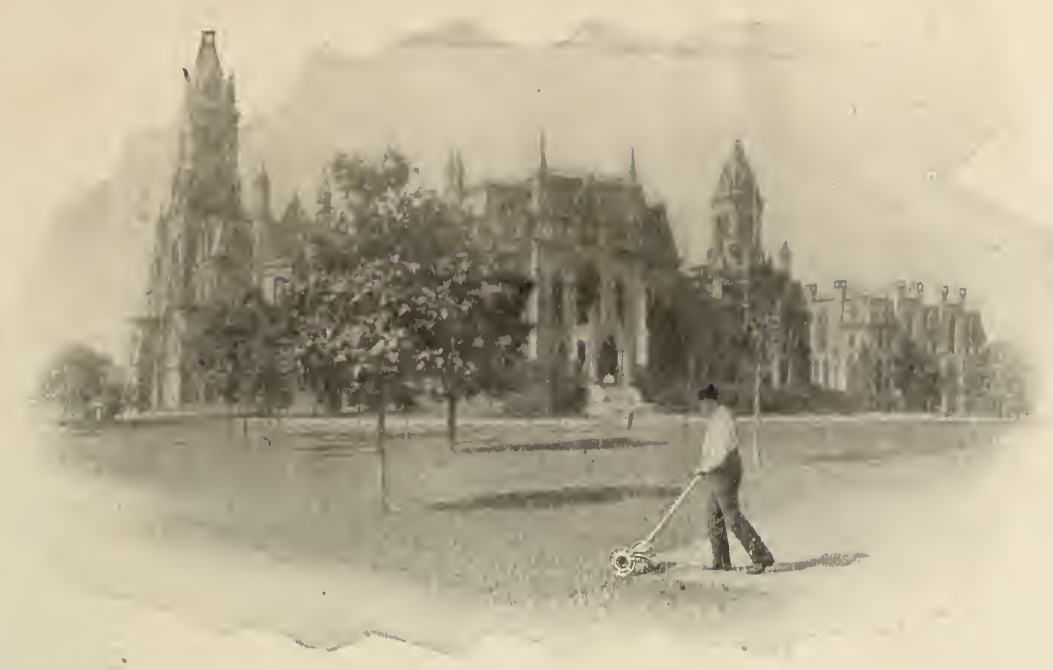

LIVINGSTON'S CAPITOL LAIWN SEED.

OUIR MIXTUIRES are composed of the purest, cleanest and heaviest Lawn Grass Seed possible to grow, and they are all carefully tested for vitality before mixing. We do our own mixing.

PRICES OF IIVINGSTON'S CAPITOL LAIWN SEED. In neat boxes for display on shelves. Large size $17 \mathrm{c}$. each, to retail @ 25c. Small size $10 \mathrm{c}$ each, to retail@15c.

KENTUCKY BLUE GRZTSS. Many prefer plain Blue Grass to a mixture, and it cannot be denied that it produces a beautiful and lasting lawn, but it takes longer to establish it. Fancy clean seed-pound, 10c., subject to market changes.

\section{DF 7 In Hardware, Groceries, Drugs, etc., wil, find Lawn Grass Seed a very neat and profitable article to handle}

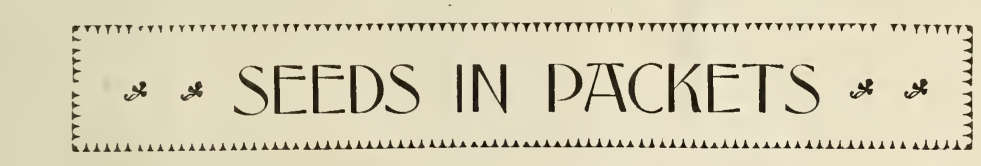

$\$ 12.00130 \%$ FOR $\$ 5.00$.

$* *$

We put up a neat box containing an assortment of the best selling kinds Vegetable Seeds all in Colored Lithograph bags, with cultural directions. Retail value $\$ 12.00$; we sell for $\$ \mathbf{5 . 0 0}$ to merchants.

\section{$* *$}

\section{CUSTOMER'S SELECTION.}

$* *$

In our seed annual we offer all kinds of vegetable and flower seeds in packets to our retail mail trade. These we furnish to our Uealers Trade at $60 \%$ discount from retail prices when $100 \mathrm{pkts}$ or more are ordered at a time. In smaller lots at $\mathbf{5 0 \%}$ discount. These packets are not to be confounded with the cheap kind usually sold. They are put up by ourselves from very best stocks and are sold by us to our retail trade. Parties who buy $100 \mathrm{pkts}$ or more at beginning of season will, of course, be entitled to later "filling in" orders at same rate. 


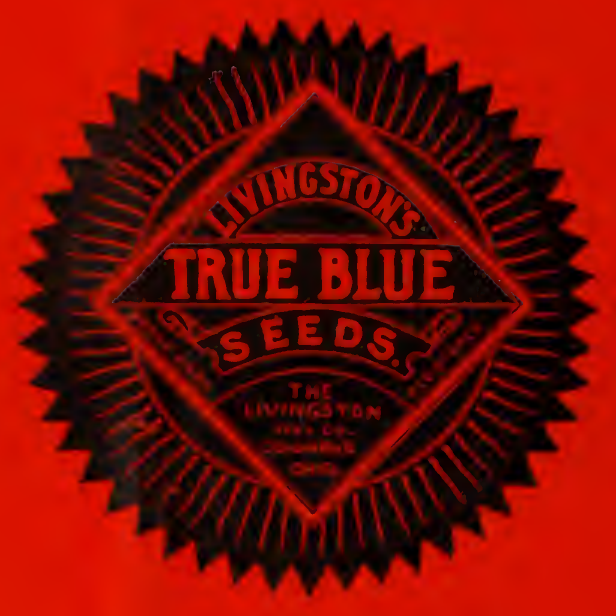

